Pacific Journal of Mathematics

COMPLETIONS OF NOETHERIAN HEREDITARY PRIM 


\section{COMPLETIONS OF NOETHERIAN HEREDITARY PRIME RINGS}

\section{K. DeshPande}

If $R$ is a Noetherian hereditary prime ring with Jacobson radical $J \neq(0)$ then, it has been shown that the $J$-adic completion of $R$ is a Noetherian hereditary semi-prime ring; it is prime if and only if the maximal ideals of $R$ form a single cycle. Among other things, one also finds the result: If $R$ is a right Noetherian semi-local ring with $\bigcap_{n=1}^{\infty} J^{n}=(0)$ then, $R$ is $J$-adic complete if and only if it is right linearly compact and $J$ has the right $A R$-property.

As is clear from the literature, it is not known whether $\hat{R}$, the $J$-adic completion of a Noetherian hereditary prime ring $R$ with $J \neq(0)$, is (right) Noetherian. It has been shown here that $\hat{R}$ is Noetherian. The approach taken proves not only this but also that $\hat{R}$ is hereditary and semi-prime. In view of Michler's structure theorem for semi-local Noetherian hereditary prime rings $[13 ; 6.5]$, the structure of $\hat{R}$ then is completely determined since, in the present case, $\hat{R}$ decomposes into prime rings each complete in its radical topology. Thus the theorem generalizes the corresponding result for Dedekind prime rings obtained in [6]. (It is immediate that if $R$ is a Noetherian semi-local ring which is either hereditary or serial then $\hat{R}$ is Noetherian, and respectively hereditary or serial.) Section 3 is concerned with the above result on HNP-rings (Hereditary Noetherian prime rings), and $\S 4$ gives a necessary and sufficient condition so that $\hat{R}$ be an HNP-ring. Section 1 gives preliminaries. Section 2 contains some general results needed for the main theorem; in particular, it contains some properties of the endomorphism ring of a quasi-injective module over a semi-local ring satisfying some additional conditions.

1. Preliminaries. All rings considered have unity, need not be commutative and the modules are unitary. $J$ denotes the Jacobson radical of a ring $R . \quad R$ is said to be semi-local if $R / J$ is Artinian. An ideal $I$ of a ring has the right $A R$-property if for each right ideal $B$ there is an $n$ such that $B \cap I^{n} \subset B I$. The socle of a module $M$ is denoted by soc $M$. The socle series of a module $M$ is the ascending sequence $\left\{\operatorname{soc}_{n} M: n \geqq 0\right\}$ of submodules of $M$ defined as follows: $\operatorname{soc}_{0} M=(0)$; $\operatorname{soc}_{n+1} M=\pi_{n}^{-1}\left(\operatorname{soc}\left(M / \operatorname{soc}_{n} M\right)\right)$ where $\pi_{n}: M \rightarrow M /$ soc $_{n} M$ is the canonical map. If $M$ is a right $R$-module and if $T$ and $N$ are subsets of $R$ and $M$ respectively, then $\operatorname{ann}_{R} N$ and 
$\operatorname{ann}_{M} T$ are defined as:

$$
\begin{aligned}
& \operatorname{ann}_{R} N=\{r \in R \mid n r=0 \quad \forall n \in N\} ; \\
& \operatorname{ann}_{M} T=\{m \in M \mid m t=0 \quad \forall t \in T\} .
\end{aligned}
$$

If $R$ is a semi-local ring with $\bigcap_{n=1}^{\infty} J^{n}=(0)$ then the $J$-adic completion $\hat{R}$ of $R$ is defined as in the commutative case. One takes for a neighborhood base of zero the set of all the powers of $J$. This defines a topology on $R$ with respect to which it is a topological ring. This topology gives rise to a metric on $R$. $\hat{R}$ is the completion of this metric space $R$ and it turns out that $\hat{R}$ is a ring containing $R$ as a subring, and is complete in its radical topology; further, $\hat{R}$ is semi-perfect. For more details one is referred to [1] and [12]. Note that $\hat{R}$ can also be realized as the inverse limit, $\lim \left(R / J^{n}\right)$, of the system $\cdots \rightarrow R / J^{3} \rightarrow R / J^{2} \rightarrow R / J$ where the maps $\overleftarrow{\text { are }}$ canonical.

Other definitions will be given whenever needed. Unexplained terms and notations will be standard and well known.

2. Some results, which are needed in the sequel, are proved in this section.

The main ideas in the proof of the following theorem may be found in $[17 ; 5.15]$. For convenience the details are given.

THEOREM 1. Let $E_{R}$ be an injective module over a ring $R$ and $S=\operatorname{End} E_{R}$, the ring of $R$-endomorphisms of $E_{R}$. If $E_{R}$ is Artinian then $S$ is left Noetherian.

Proof. Since $E$ is finite-dimensional, $S$ is semi-perfect and the radical of $S$, say $H$, consists of all $f \in S$ which annihilate essential submodules of $E$. In particular, if $f$ is in $H, \operatorname{ker} f \neq(0)$. Now let $T$ be a left ideal of $S$ contained in $H$. Suppose $A_{R}=\operatorname{ann}_{E} T=$ $\bigcap_{f \in T} \operatorname{ker} f$. Since $E$ is Artinian, the family of all submodules of $E$ of the form

$$
\operatorname{ker} f_{1} \cap \operatorname{ker} f_{2} \cap \cdots \cap \operatorname{ker} f_{n},
$$

where $f_{i} \in T$, has a minimal member. Suppose $\left({ }^{*}\right)$ is a minimal member of this family. Then for any $f \in T$, one has

$$
\operatorname{ker} f \cap \operatorname{ker} f_{1} \cap \cdots \cap \operatorname{ker} f_{n}=\operatorname{ker} f_{1} \cap \cdots \cap \operatorname{ker} f_{n} .
$$

This implies that $\operatorname{ker} f \supset \operatorname{ker} f_{1} \cap \cdots \cap \operatorname{ker} f_{n}$ and hence $A_{R}=\operatorname{ker} f_{1} \cap$ $\cdots \cap \operatorname{ker} f_{n}$.

Define $g(e)=\left(f_{1}(e), \cdots, f_{n}(e)\right)$, where $e \in E$. Then $g$ is an $R$-homomorphism of $E$ into $E \oplus E \oplus \cdots \oplus E$, the direct sum of $n$ copies of $E$. Clearly, $\operatorname{ker} g=A_{R}$. Now, let $f \in T$. Then $f(A)=(0)$ 
and $f$ and $g$ both give rise to induced mappings:

$$
\tilde{f}: E / A \longrightarrow E, \quad \tilde{g}: E / A \longrightarrow E \oplus \cdots \oplus E .
$$

Further, $\widetilde{g}$ is a monomorphism. Consider the following commutative diagram, where $\delta: E \rightarrow E / A$ is the natural map.

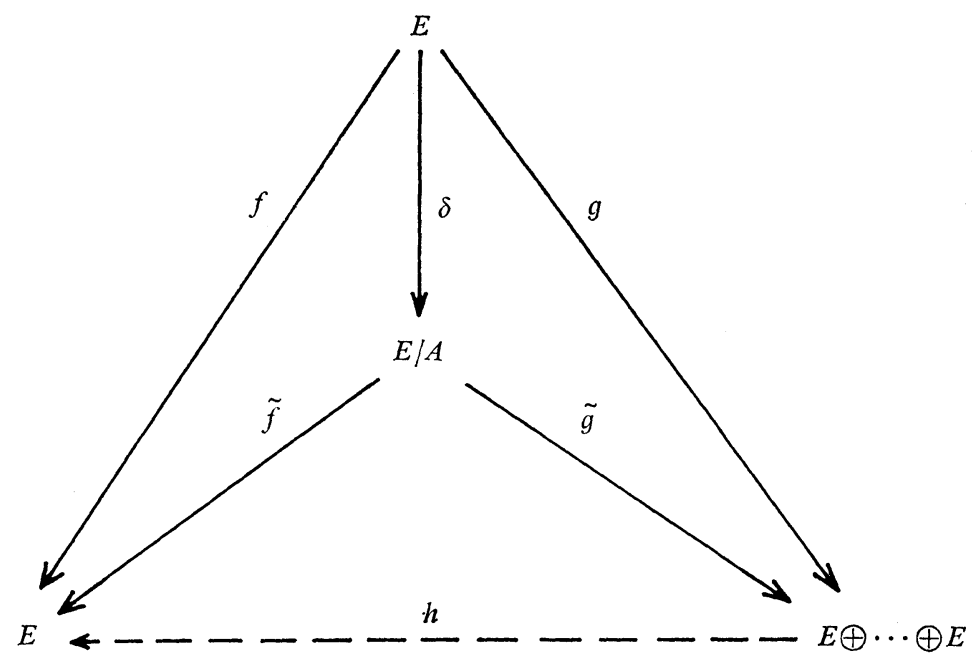

Since $E$ is injective, there is an $R$-homomorphism $h: E \oplus \cdots \oplus E \rightarrow E$, such that $h \widetilde{g}=\widetilde{f}$.

For $1 \leqq i \leqq n$, denote by $\alpha_{i}$ the $i$ th injection: $E \rightarrow E \oplus \cdots \oplus E$ and by $\pi_{i}$, the projection. Then for each $e \in E$,

$$
\begin{aligned}
f(e)=\tilde{f} \delta(e) & =h \widetilde{g} \delta(e)=h g(e) \\
& =h\left(\sum_{i=1}^{n} \alpha_{i} \pi_{i} g(e)\right)=h\left(\sum_{i=1}^{n} \alpha_{i} f_{i}(e)\right)=\sum_{i=1}^{n} h \alpha_{i} f_{i}(e) .
\end{aligned}
$$

This implies that $f=\sum_{i=1}^{n}\left(h \alpha_{i}\right) f_{i}$ and hence $T=S f_{1}+\cdots+S f_{n}$. Since $T$ is an arbitrary left ideal of $S$ contained in $H,{ }_{S} H$ is Noetherian. Since the ring $S / H$ is Artinian, it follows that $S$ is left Noetherian. This proves the theorem.

Let $M_{R}$ be a module over a ring $R,\left\{x_{i}\right\}_{i \in I} \subset M$, and $\left\{N_{i}\right\}_{i \in I}$ a set of submodules of $M$. Then the set of congruences $\left\{x \equiv x_{i} \bmod N_{i}\right\}_{I}$ is said to be solvable (finitely solvable) if there is a $y \in M$ (a $y_{F} \in M$, for each finite subset $F$ of $I$ ) such that $y-x_{i} \in N_{i}$ for each $i \in I\left(y_{F}-x_{i} \in N_{i}\right.$ for each $\left.i \in F\right) . \quad M_{R}$ is said to be Linearly Compact if every finitely solvable set of congruences in $M$ is solvable. A ring $R$ is right linearly compact if the module $R_{R}$ is linearly compact.

The following theorem establishes a connection between completeness and the linear compactness for the semi-local rings with 
$\bigcap_{n=1}^{\infty} J^{n}=(0)$.

THEOREM 2. Let $R$ be a semi-local right Noetherian ring with $\bigcap_{n=1}^{\infty} J^{n}=(0)$, where $J$ denotes the Jacobson radical of $R$. Then $R$ is $J$-adic complete if and only if it is right linearly compact and $J$ has the right AR-property.

Proof. Suppose $R$ is $J$-adic complete. By [12;2.8], $J$ has the right $A R$-property. $R$ is right linearly compact is proved by Hinohara [7; Cor. to Thm. 2].

Conversely, suppose $R$ is right linearly compact and $J$ has the right $A R$-property. By [9;4.7], the right $A R$-property for $J \mathrm{im}$ plies that $\hat{R}$ is isomorphic with the biendomorphism ring of $E_{R}$, where $E_{R}$ is the injective hull of the right $R$-module $R / J$. It is clear that $E_{R}$ is an injective cogenerator in the category of right $R$-modules. In [16; p. 342], it has been shown that a ring is right linearly compact if and only if every injective cogenerator in the category of right modules over it satisfies the doule centralizer property and is quasi-injective over its $R$-endomorphism ring. (A module $M_{R}$ has the double centralizer property means the canonical ring homomorphism of $R$ into the biendomorphism ring of $M_{R}$ is onto.) Hence, in the present case, it follows that $R \cong \hat{R}$, since $E_{R}$ is faithful. This completes the proof.

It should be noted that the above theorem is no longer true if one drops the Noetherian hypothesis. For example, a semi-primary ring is always $J$-adic complete; but a right linearly compact semiprimary ring has to be right Artinian. In a slightly different direction one can show the following: If $R$ is a semi-local ring with $\bigcap_{n=1}^{\infty} J^{n}=(0)$ such that it is $J$-adic complete, then $R$ is right Noetherian if and only if it is right linearly compact. For, if $R$ is right linearly compact then $R_{R}$ and each homomorphic image of it have finite uniform dimension [16]. Hence, if $A \neq 0$ is a right ideal of $R$, then $A \neq A J$ and $A / A J$ is finite direct sum of simple modules. Completeness of $R$ implies that $A$ is finitely generated $[12 ; 2.3$ (i)].

Some lemmas and propositions, needed later on, are given below.

LEMmA 3. Let $R$ be a semi-local ring with Jacobon radical $J$ and $M$, a right $R$-module with essential socle. If $S=$ End $M_{R}$ and if $H$ denotes the ideal of $S$ consisting of the elements which annihilate essential submodules of $M$, then for each $n$,

$$
H^{n} \subset \operatorname{ann}_{S}\left(\operatorname{ann}_{M} J^{n}\right) .
$$


If further, $M=\bigcup_{n=1}^{\infty} \operatorname{soc}_{n} M$, then $\bigcap_{n=1}^{\infty} H^{n}=(0)$.

Proof. It is well known that $H=\operatorname{ann}_{S}\left(\operatorname{soc} M_{R}\right)=\operatorname{ann}_{S}\left(\operatorname{ann}_{M} J\right)$. Suppose the statement of the lemma is true for $n$, and consider $s \in H$ and $t \in H^{n}$. Put $T_{k}=\operatorname{ann}_{M} J^{k}$. Then $s T_{n+1} J^{n} \subset s T_{1}=(0)$, since $T_{n+1} J^{n} J=(0)$. This implies $s T_{n+1} \subset T_{n}$. By hypothesis, $H^{n} \subset \operatorname{ann}_{S} T_{n}$. Thus for $t \in H^{n}$, it follows that $t T_{n}=(0)$. Hence $t s T_{n+1} \subset t T_{n}=(0)$. Since $t \in H^{n}$ and $s \in H$ are arbitrary, $H^{n+1} T_{n+1}=(0)$, that is, $H^{n+1} \subset$ $\operatorname{ann}_{S} T_{n+1}$. This proves the first statement. For the second statement note that $R$ semi-local implies $\operatorname{soc}_{n} M=\operatorname{ann}_{M} J^{n}$. Let $B_{n}=$ $\operatorname{ann}_{M} H^{n}$. By the first statement, it is clear that $B_{n} \supset \operatorname{ann}_{M} J^{n}=$ $\operatorname{soc}_{n} M$. Since $M_{R}=\bigcup_{n=1}^{\infty} \operatorname{soc}_{n} M_{R}$, it follows that ${ }_{S} M=\bigcup_{n=1}^{\infty} B_{n}$. Let $K=\bigcap_{n=1}^{\infty} H^{n}$. $K$ annihilates each $B_{n}$ and hence ${ }_{s} M$. Since ${ }_{s} M$ is faithful over $S, K=(0)$ and the lemma is proved.

Lemma 4. Let $R$ be a semi-local ring, $M_{R}$ a quasi-injective module with essential socle and $S=$ End $M_{R}$. If $M_{R}=\bigcup_{n=1}^{\infty} \operatorname{soc}_{n} M_{R}$ and if $M_{R}$ is finite-dimensional, then $\operatorname{soc}_{S} M$ is essential in ${ }_{s} M$ and ${ }_{s} M=\bigcup_{n=1}^{\infty} \operatorname{soc}_{n S} M$.

Proof. Let $H=\operatorname{rad} S$. Then $H$ consists of those elements of $S$ which annihilate essential submodules of $M_{R}$. Put $B_{n}=\operatorname{ann}_{M} H^{n}$. Since $S$ is semi-perfect, $B_{n}=\operatorname{soc}_{n S} M$. As seen in the proof of the previous lemma, $M_{R}=\bigcup_{n=1}^{\infty} \operatorname{soc}_{n} M_{R}$ implies ${ }_{S} M=\bigcup_{n=1}^{\infty} \operatorname{soc}_{n S} M$. Now, let $m \in M$. Then $m \in B_{n}$ for some $n$ and hence $H^{n} m=(0)$. Since $H$ is a two-sided ideal, $H^{n} S m=(0)$ and the cyclic submodule $S m$ of ${ }_{S} M$ is a module over the semi-primary ring $S / H^{n}$. Since every module over a semi-primary ring contains a minimal submodule, it follows that $S m$ intersects $\operatorname{soc}_{S} M$ nontrivially. (Since soc $M_{R} \neq(0)$, $\operatorname{ann}_{M} H=\operatorname{soc}_{S} M \neq(0)$.) Thus $\operatorname{soc}_{S} M$ is essential in ${ }_{S} M$.

Lemma 5. Let $M_{R}$ be a quasi-injective module over a ring $R$ and $S=$ End $M_{R}$. If $M_{R}$ has finitely generated essential socle, then ${ }_{s} M$ contains a copy of each simple left $S$-module.

Proof. It is sufficient to note that $S$ is a semi-perfect ring and $\operatorname{rad} S=\operatorname{ann}_{S}\left(\operatorname{soc} M_{R}\right)$, the soc $M_{R}$ being a left module over $S$.

Proposition 6 [9;4.3]. The following conditions on an arbitrary ring $R$ are equivalent:

(i) If $M$ is a cyclic right $R$-module with essential socle then $M=\operatorname{soc}_{n} M$ for some positive integer $n$.

(ii) If $E$ is an injective right $R$-module with essential socle then $E=\bigcup_{n=1}^{\infty} \operatorname{soc}_{n} E$. 
If $R$ is a semi-local ring then the above two conditions are equivalent to the following one:

(iii) $J$ has the right $A R$-property.

It is well known that if $T$ is a direct sum of the injective hulls of nonisomorphic copies of simple right $R$-modules then it is a (minimal) cogenerator in the category of right $R$-modules. This means every right $R$-module can be embedded in a direct product of the copies of $T$.

Proposition 7. If $R$ is an arbitrary ring for which the equivalent conditions (i) and (ii) of Prop. 6 hold then $\bigcap_{n=1}^{\infty} J^{n}=(0)$. In particular, if $R$ is a semi-local ring whose Iacobson radical $J$ has the right $A R$-property, then $\bigcap_{n=1}^{\infty} J^{n}=(0)$ even if $R$ is not right Noetherian.

Proof. Suppose $K=\bigcap_{n=1}^{\infty} J^{n} \neq(0)$. Since for each injective right $R$-module $E$ with essential socle, $E=\bigcup_{n=1}^{\infty} \operatorname{soc}_{n} E$, it is clear that $K$ annihilates injective hull of each simple right $R$-module. Consequently, $K$ annihilates the (minimal) cogenerator in the category or right $R$-modules. This implies $K$ annihilates every right $R$-module. Thus $K \neq(0)$ leads to a contradiction.

Before stating one more theorem of this section a definition is needed. For each $n=1,2, \cdots, M^{n}$ denotes a product of $n$ copies of a module $M_{R}$. If each module, which is isomorphic to a submodule of a factor module of $M^{n}$ for some $n$, can be embedded in a product of copies of $M$, then $M$ is said to be a self-cogenerator. Obviously any cogenerator in the category of $R$-modules is a selfcogenerator.

THEOREM 8. Let $R$ be a semi-local ring whose Jacobson radical $J$ has the right AR-property, $M_{R}$ a quasi-injective module with essential socle, $S=$ End $M_{R}$ and $H=\operatorname{rad} S$. Then

(i) $\bigcap_{n=1}^{\infty} H^{n}=(0)$;

(ii) if $M_{R}$ is injective and Artinian, then $S$ is a left Noetherian semi-perfect ring which is left linearly compact;

(iii) if $M_{R}$ is injective, Artinian and a self-cogenerator, then ${ }_{s} M$ is an injective cogenerator in the category of left $S$-modules and $H$ has the left AR-property; consequently, $S$ is complete in its radical topology.

Proof. (i) Since $J$ has the right $A R$-property, by Proposition $6 M_{R}=\bigcup_{n=1}^{\infty} \operatorname{soc}_{n} M_{R}$. By Lemma 3 the conclusion follows.

(ii) Since $M_{R}$ is Artinian, by Theorem $1 S$ is left Noetherian 
semi-perfect. Further, every Artinian module is linearly compact. Then as an immediate consequence of $[16 ; 3.7]$, it follows that $S$ is left linearly compact.

(iii) Since $M_{R}$ is quasi-injective, self-cogenerator and linearly compact, by [16; p. 342 Cor. 2] it follows that ${ }_{s} M$ is injective. By Lemma $5,{ }_{S} M$ contains a copy of each simple left $S$-module and hence ${ }_{S} M$ is a cogenerator in the category of left $S$-modules. Further, by Lemma $4,{ }_{S} M=\bigcup_{n=1}^{\infty} \operatorname{soc}_{n S} M$. If $N$ is a cyclic left $S$-module with essential socle, since $S$ is left Noetherian, $N$ can be embedded in a product of finite number of copies of ${ }_{s} M$. This leads to ${ }_{S} N=\operatorname{soc}_{n S} N$ for some $n$. Thus by Proposition $6, H$ has the left $A R$-property. Now, since $S$ is also left linearly compact, by Theorem $2 S$ is complete in the $H$-adic topology.

Some known examples of rings, over which the injective hull of any simple module is Artinian, are: (1) Any commutative Noetherian ring [17; 4.30]; (2) Noetherian PI-rings [10]; (3) Noetherian serial rings [8; Thm. 2], and (4) HNP-rings [18]. For all of these rings the Jacobson radical has the right, as well as left, $A R$-property. For this property of HNP-rings one may refer to [2;3.3].

3. HNP-rings. It has been shown by Chatters that any hereditary Noetherian ring $R$ satisfies the restricted minimum condition for both left and right ideals. As a consequence, the Jacobson radical $J$ of such a ring has both left and right $A R$-property. If the ring $R$ is prime with $J \neq(0)$, then $J$ is essential right ideal in $R$ and therefore $R / J$ is Artinian. Thus any HNP-ring with $J \neq(0)$ is semi-local and its radical $J$ has the $A R$-property.

In [18; Thm. 4], S. Singh shows that if $R$ is an HNP-ring which is not right primitive then the injective hull $E$ of a simple right $R$-module has a unique ascending chain of submodules

$$
(0)=x_{0} R \subset x_{1} R \subset \cdots \subset x_{n} R \subset \cdots,
$$

whose union is $E$, and the members of this chain are the only submodules of $E$. This, in particular, clearly shows that $E$ is Artinian. It has been also shown that the endomorphism ring of such a module $E$ is a local Dedekind domain which is complete (and left and right PID). With these remarks, one can have the following.

THeOREM 9. Let $R$ be an HNP-ring with $J \neq(0)$. Then the injective hull of any simple $R$-module is Artinian over its endomorphism ring.

Proof. Let $E$ be the injective hull of a simple right $R$-module. 
Then $D=$ End $E_{R}$ is a complete local domain which is both left and right PID [18; Thm. 9]. Since $D$ is an HNP-ring, injective hull of any simple $D$-module is Artinian and so is each injective $D$-module with finitely generated essential socle. Thus it is sufficient to show that ${ }_{D} E$ has finite-dimensional essential socle.

Since $E_{R}$ is Artinian and Jacobson radical of $R$ has the $A R$-property, $\operatorname{soc}_{D} E$ is essential in ${ }_{D} E$ by Lemma 4 . Now if $H$ denotes the Jacobson radical of $D$, clearly $\operatorname{ann}_{E} H$ is the socle of ${ }_{D} E$ and is a bisubmodule of ${ }_{D} E_{R}$. Since $E_{R}$ has a unique ascending chain of submodules

$$
(0)=x_{0} R \subset x_{1} R \subset \cdots \subset x_{n} R \subset \cdots,
$$

whose union is $E$, it follows that for some $m \geqq 1$, $\operatorname{soc}_{D} E=x_{m} R$. (Note that $\operatorname{soc}_{D} E$ cannot be equal to ${ }_{D} E$ since $E$ is faithful over $D$.) As $x_{m} R=\operatorname{ann}_{R} J^{m}, x_{m} R$ is an injective module over the Artinian ring $R / J^{m}$. Therefore by [5; Thm. 2], $x_{m} R$ is finitely generated over its $R / J^{m}$ - endomorphism ring, and so over its $R$-endomorphism ring. As $x_{m} R$ is fully-invariant submodule of $E_{R}$, its $R$-endomorphism ring is isomorphic to the ring $D / \operatorname{ann}_{D}\left(x_{m} R\right)$. Clearly as a module over $D / \operatorname{ann}_{D}\left(x_{m} R\right)$ and as a module over its $R$-endomorphism ring, $x_{m} R$ has the same submodule structure. Hence over $D / \operatorname{ann}_{D}\left(x_{m} R\right)$, and therefore over $D, x_{m} R$ is finitely generated left module; consequently the socle of ${ }_{D} E$ is finitely generated. This completes the proof.

Lemma 10. Let $M$ be a right module over a ring $R$ and $S=$ End $M_{R}$. Suppose $S$ contains a finite set of orthogonal idempotents $e_{1}, \cdots, e_{n}$ such that $1=e_{1}+\cdots+e_{n}$. If for each $i=1, \cdots, n, e_{i} M$ is Artinian (Noetherian) over its endomorphism ring $e_{i} S e_{i}$, then ${ }_{S} M$ is Artinian (Noetherian).

Proof. $A=e_{1} S e_{1} \oplus \cdots \oplus e_{n} S e_{n}$ is a subring of $S$ containing the unity. Also $M_{R}=e_{1} M \oplus \cdots \oplus e_{n} M$. It is easy to see that ${ }_{A} M$, and therefore ${ }_{s} M$, is Artinian (Noetherian).

One is now in a position to have the following

THEOREM 11. Let $R$ be an HNP-ring with $J \neq(0)$. Then the $J$ adic completion $\hat{R}$ of $R$ is a Noetherian hereditary semi-prime ring.

Proof. Let $E_{R}$ be an injective hull of the right $R$-module $R / J$, and $S=$ End $E_{R}$. Clearly $E_{R}$ is an injective cogenerator with finitely generated essential socle. Since $E_{R}$ is a finite direct sum of inde- 
composable injectives, each Artinian over its endomorphism ring (Theorem 9), by Lemma $10{ }_{s} E$ is Artinian. Further, $E_{R}$ itself is Artinian and a self-cogenerator. Thus by Theorem 8 (iii), ${ }_{s} E$ is an injective cogenerator in the category of left $S$-modules and $S$ is left Noetherian and complete. Now, Theorem 8 (iii) applies also to ${ }_{s} E$ and it follows that End $_{S} E$ is right Noetherian and complete in its radical topology. Since the radical $J$ of $R$ has the $A R$-property, by $[9 ; 4.7] \hat{R} \cong \operatorname{End}_{S} E$. This shows that $\hat{R}$ is right Noetherian. By symmetry, and the uniqueness of $\hat{R}$ (up to isomorphism), it is both left and right Noetherian.

Now, as $R$ is an HNP-ring, each factor module of $E_{R}$ is injective. Thus all requirements of $[14 ; 2.1]$ are satisfied by the module $E_{R}$, namely, $E_{R}$ is injective self-cogenerator and every finitely cogenerated factor of $E_{R}$ is injective. Therefore $S$ is left semi-hereditary. Since $S$ is left Noetherian, it is left hereditary. Now the same arguments hold for $S$ and ${ }_{S} E$, and hence $\hat{R} \cong \operatorname{End}_{S} E$ is right hereditary. Being Noetherian, $\hat{R}$ is both left and right hereditary.

It remains to see that $\hat{R}$ is a semi-prime ring. For this, first let $x \in R$ be a right regular element. Since $x R \cong R$ under the map $x r \rightarrow r$, it follows that $x \hat{R} \cong \hat{R}$ under the map $x \hat{r} \rightarrow \hat{r}$ (see the proof of $[6 ; 3.4])$. Thus $x$ is also a right regular element in $\hat{R}$. In particular, every regular element of $R$ remains regular in $\hat{R}$.

Now as $\hat{R}$ is a Noetherian hereditary ring, by a well known theorem of Chatters [3], $\hat{R}=A \oplus B$ where $A$ is an Artinian and $B$ a semi-prime ring. Since $R$ is prime, $J$ is essential in $R$ and hence contains a regular element $t$. Therefore $t$ is regular in $\hat{R}$ and is contained in its Jacobson radical $\hat{J}$. This implies that $\hat{R}$ cannot have a minimal left or right ideal. Thus $A=(0)$ and $\hat{R}$ is semiprime.

CoRollary 13. Let $R$ be an HNP-ring with $J \neq(0)$ and $M_{R}$ an injective cogenerator with finitely generated essential socle. The endomorphism ring of $M_{R}$ is left hereditary, left Noetherian and complete in its radical topology.

COROLlaRY 14. If $R$ is an hereditary Noetherian semi-local ring with Jacobson radical $J$, then the J-adic completion $\hat{R}$ is a product of an Artinian ring and a finite number of HNP-rings, each complete in its radical topology.

Proof. Since $R$ is Noetherian and hereditary, by the theorem of Chatters [3], $R=A \oplus B_{1} \oplus \cdots \oplus B_{n}$, where $A$ is an Artinian ring and each $B_{i}$ is an HNP-ring. It is easy to verify that $\hat{R}=$ $A \oplus \hat{B}_{1} \oplus \cdots \oplus \hat{B}_{n}$, where $\hat{B}_{i}$ is the $J$-adic completion of $B_{i}$. Each 
$\widehat{B}_{i}$ is a Noetherian hereditary complete semi-prime ring and will decompose as a direct product of HNP-rings, each complete in its radical topology.

In [20; 5.11], Warfield has shown that if $R$ is a Noetherian serial ring, with Jacobson radical $J$, then $\bigcap_{n=1}^{\infty} J^{n}=0$, and $R$ is the product of an Artinian serial ring and a hereditary serial ring with no simple left or right ideals. Clearly the latter type of serial ring is semi-prime; (see the proof of $[20 ; 5.14]$ ). Moreover, by [20; 4.7] any semi-perfect, semi-prime Noetherian ring is hereditary if and only if it is a serial ring. Recalling that any complete semilocal ring is semi-perfect, one obtains the following

CoROLlaRY 15. Let $R$ be a Noetherian serial ring with Jacobson radical $J$. Then the J-adic completion of $R$ is also a Noetherian serial ring.

4. Theorem 11 states that if $R$ is an HNP-ring with $J \neq(0)$, then the $J$-adic completion $\hat{R}$ of $R$ is a hereditary Noetherian semiprime ring. One may obviously sak: "Why isn't $\hat{R}$ prime?"

Firstly, it can be easily seen that, in general, $\hat{R}$ need not be prime. For this, let $S$ be a commutative Dedekind domain, say the ring of integers, and $P_{1}, P_{2}, \cdots, P_{m}$ a finite number of nonzero prime ideals of $S$; (obviously $P_{i}$ are maximal). Let $M$ be the complement of the union of $P_{1}, \cdots, P_{m}$. The quotient ring $S_{M}$, with respect to the multiplicative system $M$, is again a Dedeking domain with exactly $m$ maximal ideals [21; Example 2, p. 227, Example 2, p. 270]. This ring $S_{M}$ serves the purpose; (when $S$ is the ring of integers and $p_{i}$ prime numbers such that $P_{i}=\left(p_{i}\right), S_{M}$ is the ring consisting of rationals with denominators not divisible by any of the $p_{i}$ ). Set $R=S_{M}$, and let $M_{1}, \cdots, M_{m}$ be the maximal ideals of $R$; then its Jacobson radical $J=M_{1} \cap \cdots \cap M_{m}=M_{1} M_{2} \cdots M_{m}$. In general, $J^{n}=$ $M_{1}^{n} \cap M_{2}^{n} \cap \cdots \cap M_{m}^{n}$. This leads to $R / J^{n} \cong R / M_{1}^{n} \oplus \cdots \oplus R / M_{m}^{n}$, hence $\lim \left(R / J^{n}\right) \cong \lim \left(R / M_{1}^{n}\right) \oplus \cdots \oplus \lim \left(R / M_{m}^{n}\right)$. Thus $\hat{R}$ is not indecomposable, and so cannot be a $\overleftarrow{\text { prime ring. }}$

For describing conditions intrinsic to $R$, which render $\hat{R}$ of Theorem 11 prime or not prime, a theorem of B.J. Muller is helpful. Before stating this theorem, some definitions are needed.

Let $R$ be a right Noetherian ring, $S=\bigcap_{i=1}^{n} P_{i}$ a semi-prime ideal, where the prime ideals $\left\{P_{1}, \ldots, P_{n}\right\}$ are supposed to be incomparable. $S$ is called right-localizable if the multiplicative set $\mathscr{C}(S)=$ $\{c \in R \mid c x \in S$ implies $x \in S\}$ is right Ore. Let $\rho_{S}$ denote the left exact radical corresponding to the torsion theory determined by $\mathscr{C}(S)$. $\rho_{S}(R)$ is an ideal of $R$. The image $\overline{\mathscr{C}(S)}$ of $\mathscr{C}(S)$ under the canonical map $R \rightarrow \bar{R}=R / \rho_{S}(R)$ is a regular right Ore set in $\bar{R}$ when 
$\mathscr{C}(S)$ is right Ore in $R$. The (classical) right quotient ring of $\bar{R}$ w.r.t. $\overline{\mathscr{C}(S)}$ is defined to be the right quotient ring of $R$ at the semi-prime ideal $S$, and is denoted by $R_{S}$. $R_{S}$ happens to be a right Noetherian semi-local ring (when $S$ is right-localizable).

$S$ is called right-classical if it is right-localizable and if, in addition, the Jacobson radical of $R_{S}$ has the right $A R$-property.

A ring $R$ is Noetherian, means it is left and right Noetherian; the same applies to the terms like localizable, classical etc.

A nonempty finite set $\left\{P_{1}, \ldots, P_{n}\right\}$ of mutually incomparable prime ideals is called a Clan if its associated semi-prime ideal $\bigcap_{i=1}^{n} P_{i}$ is classical but the associated semi-prime ideals of all proper subsets are not.

Now the Muller's theorem may be stated.

TheOREM 16 [15; Thm. 4]. Let $R$ be a Noetherian ring with the classical semi-prime ideal $S=\bigcap_{i=1}^{n} P_{i}$. Then there is a one-toone correspondence between the central idempotents of $\hat{R}_{S}$ and the localizable subsets of $\left\{P_{1}, \cdots, P_{n}\right\}$ (where $\hat{R}_{S}$ denotes the $J\left(R_{S}\right)$-adic completion of $\left.R_{S}\right)$. Such subsets are automatically classical.

Since $\hat{R}_{S}$ is semi-perfect, Theorem 16 implies that $\left\{P_{1}, \ldots, P_{n}\right\}$ is uniquely partitioned into clans, and clans correspond to centrally indecomposable central orthogonal idempotents of $\hat{R}_{S}$. For these results, and the above preliminaries, one may refer to $[15,9]$.

Now coming to the main objective of this section, first note that if $R$ is an HNP-ring with $J \neq(0)$, then its maximal ideals fall into disjoint cycles [4]; a clan of maximal ideals is a cycle and vice versa $[15 ; \S 3.3]$. Further, for any semi-local ring $R$ with Jacobson radical $J, \mathscr{C}(J)$ consists of the units of $R ; J$ is localizable, and ${ }_{J} R=R_{J}=R$. Thus, in Theorem 16 , if $R$ is also a semi-local ring and $S$ its radical then $\hat{R}$ and $\hat{R}_{S}$ coincide. The following theorem is now obvious.

THEOREM 17. Let $R$ be an HNP-ring with $J \neq(0)$, and $\hat{R}$ the $J$-adic completion of $R$. The hereditary Noetherian semi-prime ring $\hat{R}$ decomposes into $n$ prime rings if and only if the maximal ideals of $R$ fall into $n$ distinct cycles.

COROLLARY 18. Let $R$ be an HNP-ring with $J \neq(0)$, then the completion $\hat{R}$ is also an HNP-ring if and only if the maximal ideals of $R$ form a single cycle.

REMARKs. (i) The referee has brought to the author's notice a recent paper of $P$. Vamos [19] in which, among other things, it 
has been shown that if $R$ is a semi-local ring satisfying a polynomial identity, then $\hat{R}$ is a Noetherian semi-local PI-ring satisfying all the identities of $R$, and $\hat{R}$ has Morita duality.

If $R$ is an HNP-ring with $J \neq(0)$, by Theorem $2, \hat{R}$ is (both left and right) linearly compact. Since $\hat{R}$ is a direct sum of HNPrings, it is easy to see that the $\hat{R}$-injective hull of $\hat{R} / \hat{J}$ is Artinian. Thus, by [16; Cor 2, p. 344], $\hat{R}$ has Morita duality in this case also. In fact, any complete Noetherian semi-local ring whose minimal injective cogenerator is Artinian, has Morita duality.

(ii) The present paper lists commutative Noetherian rings, Noetherian PI-rings, Noetherian serial rings and HNP-rings as the known classes of rings over which the injective hull of any simple module is Artinian. As is now clear, the completion of a Noetherian semi-local ring belonging to any of the above classes is Noetherian. Hence one would be tempted to ask the following question (which will be left as open):

If $R$ is a Noetherian semi-local ring over which the injective hull of any simple module is Artinian, is $\hat{R}$ Noetherian?

Note that for such a ring $R$, the Jacobson radical $J$ has the $A R$-property.

(iii) In [11], it has been shown that if $M$ is a maximal ideal of a Dedekind prime ring $R$ and $P$ is any maximal right ideal containing $M$, then the $M$-adic completion $\hat{R}$ of $R$ can be realized as the biendomorphism ring of the injective hull of the right $R$-module $R / P$ (and $\hat{R}$ is a local principal ideal domain). In view of $[9 ; 4.7]$ and [13], this result generalizes, with appropriate modifications, to the case where $R$ is an HNP-ring and $M$ its Jacobson radical. An obvious question is: Can same thing be said when $R$ is an arbitrays HNP-ring and $M$ any invertible ideal?

The author proposes to further exploit Theorem 16 in a separate communication; as a consequence of the results ontained there, one will find an affirmative answer to the above question.

ACKNOWLEDGMENTS. The author also wishes to thank the referee of this paper, who saved him from inaccuracies, and whose valuable suggestions helped improve the paper.

\section{REFERENCES}

1. E. H. Batho, Non-commutative semi-local rings, Duke Math. J., 24 (1957), 163-172.

2. A. W. Chatters, The restricted minimum condition in Noetherian hereditary rings, J. London Math. Soc., (2), 4 (1971), 83-87.

3. — A decomposition theorem for Noetherian hereditary rings, Bull. London Math. Soc., 4 (1972), 125-126.

4. D. Eisenbud and J.C. Robson, Hereditary Noetherian prime rings, J. Algebra, 16 
(1970), 86-104.

5. C. Faith, Modules finite over endomorphism ring, Lectures on rings and Modules, 246 Springer-Verlag, (1972), 145-189.

6. W.D. Gwynne and J.C. Robson, Completions of non-commutative Dedekind prime rings, J. London Math. Soc., (2), 4 (1971), 346-352.

7. Y. Hinohara, Note on non-commutative semi-local rings, Nagoya Math. J., 17 (1960), 161-166.

8. G. Ivanov, Decomposition of modules over serial rings, Comm. in Algebra, 3 (11) (1975), 1031-1036.

9. A. V. Jategaonkar, Injective modules and localization in non-commutative Noetherian rings, Trans. Amer. Math. Soc., 190 (1974), 109-123.

10. Certain injectives are Artinian, Lecture notes in Math., 545, SpringerVerlag (1976), 128-139.

11. J. Kuzmanovich, Completions of Dedekind prime rings as second endomorphism rings, Pacific J. Math., 36 (1971), 721-729.

12. J. C. McConnell, The Noetherian property in complete rings and modules, J. Algebra, 12 (1969), 143-153.

13. G. Michler, Structure of semi-perfert hereditary Noetherian ringd, J. Algebra, 13 (1969), 327-344.

14. R. W. Miller and D. R. Turnidge, Factors of co-finitely generated injective modules,

Comm. in Algebra, 4 (3), (1976), 233-243.

15. B. J. Muller, Localization in non-commutative Noetherian rings, Canad. J. Math., 28 (1976), 600-610.

16. F. L Sandomierski, Linearly compct modules and local Morita duality, Ring Theory (Ed., R. Gordon), Academic Press (1972), 333-346.

17. D. W. Sharpe and P. Vamos, Injective Modules, Camb. Univ. Press, 1972.

18. S. Singh, Quasi-injective and quasi-projective modules over HNP-rings, Canad. J. Math., 26 (1974), 1133-1185.

19. P. Vamos, Semi-local Noetherian PI-rings, Bull. London Math. Soc., 9 (1977), 251-256.

20. R. B. Warfield, Jr., Serial rings and finitely presented modules, J. Algebra, 37 (1975), $187-222$.

21. O. Zariski and P. Samuel, Commutative Algebra I, Van Nostrand, 1962.

Received July 18, 1978 and in revised form September 26, 1979. The main part of this paper is based on the author's Ph. D. dissertation at the University of Wisconsin, Milwaukee. The author wishes to express his appreciation to his supervisor Professor Ann K. Boyle for innumerable suggestions and the encouragement.

Marathwada University

Aurangabad 431004

(Maharashtra) INDIA 



\section{PACIFIC JOURNAL OF MATHEMATICS}

\section{EDITORS}

DONALD BABBITT (Managing Editor)

University of California

Los Angeles, CA 90024

Hugo RossI

University of Utah

Salt Lake City, UT 84112

C. C. MOORE and ANDREW OGG

University of California

Berkeley, CA 94720

\section{J. DugunduI}

Department of Mathematics

University of Southern California

Los Angeles, CA 90007

R. Finn and J. Milgram

Stanford University

Stanford, CA 94305

\section{ASSOCIATE EDITORS}
E. F. BeCKenBACH
B. H. NeumanN
F. WOLF
K. YosHIDA

\section{SUPPORTING INSTITUTIONS}

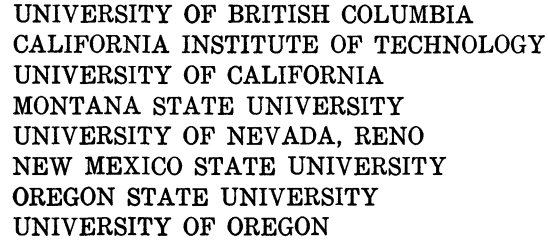

UNIVERSITY OF BRITISH COLUMBIA CALIFORNIA INSTITUTE OF TECHNOLOGY UNIVERSITY OF CALIFORNIA

MONTANA STATE UNIVERSITY

UNIVERSITY OF NEVADA, RENO

NEW MEXICO STATE UNIVERSITY

OREGON STATE UNIVERSITY UNIVERSITY OF OREGON

\author{
UNIVERSITY OF SOUTHERN CALIFORNIA \\ STANFORD UNIVERSITY \\ UNIVERSITY OF HAWAII \\ UNIVERSITY OF TOKYO \\ UNIVERSITY OF UTAH \\ WASHINGTON STATE UNIVERSITY \\ UNIVERSITY OF WASHINGTON
}

The Supporting Institutions listed above contribute to the cost of publication of this Journal, but they are not owners or publishers and have no responsibility for its content or policies.

Mathematical papers intended for publication in the Pacific Journal of Mathematics should be in typed form or offset-reproduced, (not dittoed), double spaced with large margins. Please do not use built up fractions in the text of the manuscript. However, you may use them in the displayed equations. Underline Greek letters in red, German in green, and script in blue. The first paragraph or two must be capable of being used separately as a synopsis of the entire paper. Please propose a heading for the odd numbered pages of less than 35 characters. Manuscripts, in triplicate, may be sent to any one of the editors. Please classify according to the scheme of Math. Reviews, Index to Vol. 39. Supply name and address of author to whom proofs should be sent. All other communications should be addressed to the managing editor, or Elaine Barth, University of California, Los Angeles, California, 90024.

50 reprints to each author are provided free for each article, only if page charges have been substantially paid. Additional copies may be obtained at cost in multiples of 50 .

The Pacific Journal of Mathematics is issued monthly as of January 1966. Regular subscription rate: $\$ 84.00$ a year (6 Vols., 12 issues). Special rato: $\$ 42.00$ a year to individual members of supporting institutions.

Subscriptions, orders for numbers issued in the last three calendar years, and changes of address shoud be sent to Pacific Journal of Mathematics, P.O. Box 969, Carmel Valley, CA 93924, U.S.A Old back numbers obtainable from Kraus Periodicals Co., Route 100, Millwood, NY 10546.

PUBLISHED BY PACIFIC JOURNAL OF MATHEMATICS, A NON-PROFIT CORPORATION

Printed at Kokusai Bunken Insatsusha (International Academic Printing Co., Ltd.). 8-8, 3-chome, Takadanobaba, Shinjuku-ku, Tokyo 160, Japan.

Copyright (C) 1980 by Pacific Jounal of Mathematics Manufactured and first issued in Japan 


\section{Pacific Journal of Mathematics \\ Vol. 90, No. $2 \quad$ October, 1980}

Frank Hayne Beatrous, Jr., Hölder estimates for the $\bar{\partial}$ equation with a support condition ..................................... 249

Charles L. Belna, Michael Jon Evans and Paul Humke, Planar continua

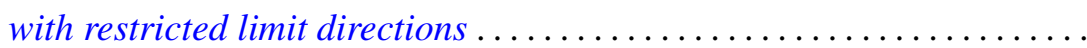

Leon Brown and Takashi Ito, Classes of Banach spaces with unique isometric preduals................................. 261

V. K. Deshpande, Completions of Noetherian hereditary prime rings ..... . 285

Deepak Dhar, Asymptotic enumeration of partially ordered sets . . . . . . . 299

Zeev Ditzian, On interpolation of $L_{p}[a, b]$ and weighted Sobolev

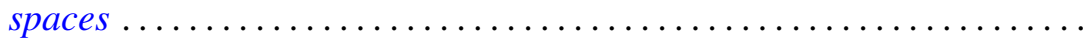

Andrew George Earnest, Congruence conditions on integers represented by

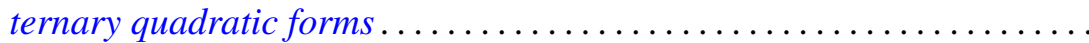

Melvin Faierman, Bounds for the eigenfunctions of a two-parameter system of ordinary differential equations of the second order ..............

Hector O. Fattorini, Vector-valued distributions having a smooth

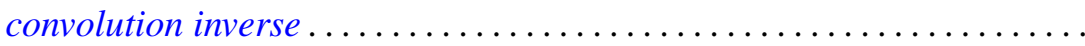

Howard D. Fegan, The spectrum of the Laplacian on forms over a Lie

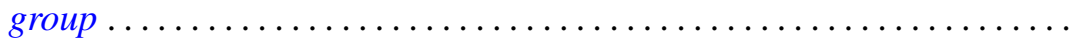

Gerald Leonard Gordon, On the degeneracy of a spectral sequence associated to normal crossings..

S. Madhavan, On bisimple weakly inverse semigroups ... 397

Françoise Mathot, On the decomposition of states of some

Roger McCann, Embedding asymptotically stable dynamical systems into radial flows in $l_{2}$

Michael L. Mihalik, Ends of fundamental groups in shape and proper homotopy...............................

Samuel Murray Rankin, III, Boundary value problems for partial functional differential equations .

Randy Tuler, Arithmetic sums that determine linear characters on $\Gamma(N)$

Jeffrey D. Vaaler, On linear forms and Diophantine approximation ..

G. P. Wene, Alternative rings whose symmetric elements are nilpotent or a right multiple is a symmetric idempotent. . 